\title{
ORIGINAL ARTICLE \\ Retrospective assessment of the validity and use of the community balance and mobility scale among individuals with subacute spinal cord injury
}

\author{
$\mathrm{K}_{\text {Chan }}{ }^{1}$, K Guy ${ }^{1,2}$, G Shah ${ }^{1,3}$, J Golla ${ }^{1}$, HM Flett ${ }^{2,4}$, J Williams ${ }^{2,4}$ and KE Musselman ${ }^{1,4}$
}

\begin{abstract}
Study design: This is a retrospective chart review.
Objectives: The objective of this study was to evaluate the convergent validity, internal consistency and use of the Community Balance \& Mobility Scale (CB\&M) in individuals with incomplete spinal cord injury (iSCl) during inpatient rehabilitation.

Setting: This study was conducted in an $\mathrm{SCl}$ rehabilitation center in Canada.

Methods: Inpatient charts from January 2009 to October 2015 were screened. A chart was excluded if the inpatient was aged $>65$ years, did not complete a CB\&M and/or had a comorbid condition. Demographics, CB\&M score and injury-related characteristics were extracted. Berg Balance Scale (BBS), Six-Minute Walk Test (6 MWT) and Ten-Meter Walk Test (10 mWT) scores were obtained if completed within 1 week of the CB\&M. $\chi^{2}$-Tests were used to identify CB\&M items that were uniformly distributed, implying good discrimination between individuals. Convergent validity was assessed by correlating (Pearson's $r$ ) CB\&M scores with BBS, 6 MWT and 10 mWT scores. Internal consistency (Cronbach's $\alpha$ ) was evaluated.

Results: Thirty inpatients were included (23 male, 38.3 \pm 15.3 years old, three AIS C, 26 AIS D, C1-L4, 16 traumatic). BBS scores were $\geqslant 51 / 56$; however, CB\&M scores showed a wider range (27-82/96). Unilateral Stance, Tandem Walking, Lateral Foot Scooting, Forward to Backward Walking and Descending Stairs showed uniform distributions. Scores on the CB\&M correlated strongly with scores on the $6 \mathrm{MWT}(r=0.72, P<0.001)$ and moderately strongly with $10 \mathrm{mWT}$ and BBS scores $(r=0.47-0.59, P=0.004-0.013)$. Cronbach's $\alpha=0.87$.

Conclusions: The CB\&M is a valid measure in high-functioning individuals with iSCI. Prospective studies are required to further evaluate the psychometric properties of the CB\&M as a measure of high-level balance for SCl.
\end{abstract}

Spinal Cord (2017) 55, 294-299; doi:10.1038/sc.2016.140; published online 27 September 2016

\section{INTRODUCTION}

Regaining the ability to walk is an important goal for individuals with incomplete spinal cord injury (iSCI) ${ }^{1,2}$ More specifically, achieving a level of walking ability that is functional, safe and effective is of high importance. ${ }^{1}$ Balance, defined here as the ability to keep one's center of mass within one's base of support, is an essential component of walking in real-world environments that may be compromised after sustaining an SCI. Limited balance control unavoidably affects walking ability, as balance is considered a major determinant of walking status and performance. ${ }^{1,3}$ Intact balance strategies are essential to minimize the risk of falls that, in the case of individuals with iSCI, most commonly occur while walking. ${ }^{4-6}$ Up to $75 \%$ of those with iSCI fall each year, ${ }^{4-6}$ leading to injuries and hospital admissions that negatively affect the quality of life of those with iSCI ${ }^{4}$ and increase the healthcare costs. $^{7,8}$

The Berg Balance Scale (BBS) is the only balance measure that has been widely used across the SCI population. It is a 14-item objective measure designed to assess static balance and fall risk in elderly populations. ${ }^{9}$ The BBS has been deemed a valid clinical measure for individuals with iSCI. ${ }^{10,11}$ Unfortunately, the BBS has a ceiling effect, which means that it cannot distinguish between higher-functioning individuals with iSCI who have differing ambulatory balance capabilities. ${ }^{11}$ In addition, achieving a high score on the BBS does not translate to high walking function among individuals with SCI. ${ }^{12}$ This is not surprising given that the BBS consists of sitting and standing, but not walking, activities.

As most falls among individuals with iSCI occur while walking, ${ }^{7}$ a measure of high-level balance with an emphasis on walking is needed for the iSCI population. Such a scale was previously developed for the brain-injured population: the Community Balance \& Mobility Scale $(\mathrm{CB} \& M)$ is a 19-item scale that measures performance of challenging balance and mobility tasks. ${ }^{13}$ The tasks require speed, precision and accuracy, and reflect motor skills needed for function and community participation. ${ }^{13}$ For example, items on the CB\&M include Tandem Walking, Lateral Foot Scooting, Walking and Looking, and Running with a Controlled Stop. Each item is scored on a 5- or 6-point ordinal

${ }^{1} \mathrm{SCl}$ Mobility Lab, Toronto Rehabilitation Institute—University Health Network (TRI-UHN), Toronto, Ontario, Canada; ${ }^{2}$ Brain \& Spinal Cord Rehabilitation Program, Toronto Rehabilitation Institute-University Health Network TRI-UHN, Toronto, Ontario, Canada; ${ }^{3}$ Department of Neuroscience, University of Toronto, Toronto, Ontario, Canada and ${ }^{4}$ Department of Physical Therapy, University of Toronto, Toronto, Ontario, Canada

Correspondence: Dr KE Musselman, SCI Mobility Lab, Toronto Rehabilitation Institute_-University Health Network (TRI-UHN), 520 Sutherland Dr., Toronto, Ontario, Canada M4G $3 \mathrm{~V} 9$.

E-mail: Kristin.Musselman@uhn.ca

Received 2 June 2016; revised 15 August 2016; accepted 22 August 2016; published online 27 September 2016 
scale ( 0 to 5 ; one item 0 to 6 ), with higher scores indicating better performance. The CB\&M has been validated in traumatic brain injury ${ }^{13}$ and used in the following populations: pediatric acquired brain injury, ${ }^{14}$ older adults, ${ }^{15}$ children, ${ }^{16}$ stroke, ${ }^{17}$ individuals with knee osteoarthritis ${ }^{18}$ and hemophilia. ${ }^{19}$ Although not yet documented in the literature, the CB\&M has also been used in individuals with iSCI at our rehabilitation facility. We had the opportunity to perform a retrospective assessment of the use of the CB\&M among individuals with subacute SCI. ${ }^{11}$ The objectives of this study were to (1) evaluate the convergent validity and internal consistency of the CB\&M and (2) describe the use of the CB\&M among physiotherapists during inpatient SCI rehabilitation. For example, it was important to understand when during inpatient rehabilitation the measure was administered, and to which individuals with SCI. We hypothesized that the CB\&M would (1) demonstrate convergent validity as evidenced by moderately strong correlations between scores on the $\mathrm{CB} \& \mathrm{M}$ and measures of walking ability, (2) show high internal consistency and (3) be used mainly in high-functioning individuals with AIS D SCI.

\section{MATERIALS AND METHODS}

A retrospective chart review was performed at Canada's largest SCI rehabilitation center. Ethical approval was obtained from the Research Ethics Board of the University Health Network. Inpatient charts spanning the time period 1 January 2009 to 31 October 2015 were screened. First, only charts with a minimum score of 115 points (out of a possible 126) on the discharge Functional Independence Measure were retrieved (that is, walking was the main means of mobility). This was done to ensure that only the charts of those capable of completing the CB\&M were screened. These charts were then screened by one researcher $(\mathrm{KC})$. Individuals with SCI were included if they were (1) 65 years of age or younger, and (2) assessed on the CB\&M and one other balance or walking measure (BBS, $10 \mathrm{mWT}$ or $6 \mathrm{MWT}$ ) within 1 week of each other. The $6 \mathrm{MWT}$ assesses the distance walked (in meters) over $6 \mathrm{~min},{ }^{20}$ whereas the $10 \mathrm{mWT}$ assesses walking speed in meters per second. The $10 \mathrm{mWT}$ can be performed at a self-selected (SS; that is, comfortable) walking speed or at a fast speed (FS; $10 \mathrm{mWT}$ SS and $10 \mathrm{mWT}$ FS, respectively). Both the $10 \mathrm{mWT}$ and $6 \mathrm{MWT}$ have been proven valid and reliable walking measures for the iSCI population. ${ }^{21}$ Participants were excluded if they had a significant comorbid condition (for example, brain injury, multiple sclerosis, vestibular disorder).

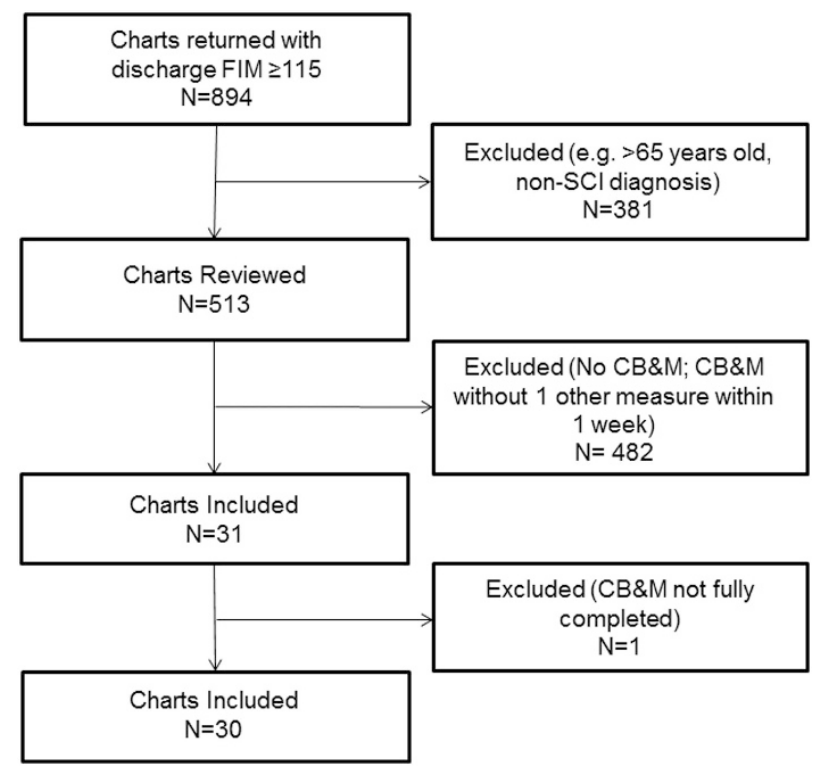

Figure 1 Flowchart of chart inclusion and exclusion.

\section{Data extraction}

The following demographic and injury-related characteristics were extracted from the charts of all included participants: age, gender, American Spinal Injury Association Impairment Scale score (AIS), lower extremity motor score (LEMS), neurological level of injury, length of inpatient stay and number of falls experienced as an inpatient. We also extracted the dates and scores of the CB\&M, BBS, 6 MWT and $10 \mathrm{mWT}$, when available. For the CB\&M, scores on each of the 19 test items were extracted, as well as the total score. If more than one CB\&M was completed for a participant during their inpatient stay, we extracted the information from the CB\&M that was accompanied by a $10 \mathrm{mWT}, 6 \mathrm{MWT}$ and/or BBS. Extracted data were entered into a single extraction table (Excel, Microsoft).

\section{Data analysis}

Descriptive statistics, such as means \pm s.d. and range of scores, were used to describe participant characteristics. To compare the performance on the BBS and the CB\&M within participants, the total scores were expressed as percentages (that is, $(\mathrm{BBS}$ score $/ 56 \times 100 \%)$ and $(\mathrm{CB} \& \mathrm{M}$ score $/ 96 \times 100 \%))$ and then plotted. As all participants achieved high scores on the BBS $(\geqslant 51 / 56$, see Results), we wanted to determine whether any items on the CB\&M were able to discriminate between participants. To do this, the $\chi^{2}$-test was used to determine whether the distribution of scores for each CB\&M test item was uniform or not. Items with uniform distributions (that is, nonsignificant $\chi^{2}$-test result) were deemed discriminatory items, as participants' scores were spread approximately evenly across the possible scores ( 0 to 5 or 6$)$. To evaluate the convergent validity of the CB\&M, we correlated scores on the CB\&M with scores on the 6 MWT, $10 \mathrm{mWT}$ FS, $10 \mathrm{mWT}$ SS and BBS using Pearson's correlation coefficient $(r)$. The strength of the correlation was determined by the magnitude of the correlation coefficient: the correlation was weak if $0<|r|$ $<0.4$, moderately strong if $0.4<|r|<0.6$ and strong if $0.6<|r|{ }^{22}$ To evaluate internal consistency, Cronbach $\alpha$ was used. A Cronbach $\alpha$ in the range of $0.7-0.95$ would suggest high internal consistency without redundancy of test items. ${ }^{23}$ Alpha was set to 0.05 for all statistical tests, which were performed with IBM SPSS 23 (IBM Corp., Armonk, NY, USA).

\section{RESULTS}

\section{Participants}

A total of 894 charts were identified as belonging to inpatients who scored $\geqslant 115$ on the Functional Independence Measure during the 6.8-year period studied. After filtering the charts for the excluded diagnoses and age $>65$ years, 513 charts remained for review (Figure 1). Of these 513 charts, 31 inpatients met all inclusion criteria. All CB\&M assessments were administered and scored by a physical therapist.

One inpatient was subsequently excluded because the CB\&M assessment was only partially completed before discharge, resulting in 30 participants ( 23 males) for analysis. Two additional participants were unable to complete some CB\&M items because of the nature of their SCI or the type of external fixation used (for example, halo). In these participants, some of the test items were considered too risky to perform and/or the participant was unable to turn his/her head or trunk to complete some items, and thus a score of 0 was assigned by the physical therapist. These two participants were not excluded from the analysis because all CB\&M items were scored. Furthermore, the inclusion of their data gives a realistic reflection of the use and feasibility of the CB\&M in SCI.

The mean age of the participants was $38.3 \pm 15.3$ years (range: 16-64 years). Upon admission to inpatient rehabilitation, 26 subjects were AIS D, three were AIS C and one did not have an AIS score reported in his/her chart. The neurological level of injury ranged from C1 to L4 (17 cervical, six thoracic and five lumbar). The specific neurological level of injury was not reported in the charts of two participants. Sixteen participants had injuries that were considered 
traumatic, whereas 14 had nontraumatic SCI (three due to a benign tumor, two due to a malignant tumor, four due to spondylosis, two due to a hemorrhage affecting the spinal cord and one due to each of transverse myelitis, syringomeylia and an intraspinal abscess). The average LEMS of the participants on admission was $43.7 \pm 8.6$ (range: 18-50). The average length of stay for inpatient rehabilitation was $41.7 \pm 24.6$ days (range: $6-122$ days). The CB\&M was administered an average of $33.2 \pm 28.2$ days post admission to inpatient rehabilitation (range: 2-121 days). Two participants experienced one fall during their inpatient stay. These participants were 26 and 38 years old, had AIS levels C and D and CB\&M scores of 56 and 52, respectively.

\section{The BBS and CB\&M}

We compared scores on the BBS with the CB\&M scores for 27 participants; three were excluded from this analysis because they completed the BBS $>1$ week before the CB\&M. In all but two participants, the CB\&M was performed after the BBS had been administered. All participants scored $\geqslant 51$ on the BBS (mean $54.5 \pm 1.6$, range 51-56), meaning that all participants achieved a near-perfect score. In contrast, scores on the CB\&M were dispersed (mean 51.4 \pm 14.1, range 27-82; Figure 2). Interestingly, a higher score on the BBS did not always translate to a higher score on the CB\&M. One participant who received a score of 53 on the BBS achieved a higher CB\&M score (82) compared with all other participants who scored a 54,55 or 56 on the BBS.

\section{Uniformity of CB\&M test items}

Six items on the CB\&M showed a uniform distribution of scores across participants $\left(\chi^{2}\right.$-test, $\left.P>0.05\right)$, suggesting that they may be particularly useful for discriminating between individuals with SCI with higher levels of function. The items were as follows: Unilateral Stance on the Right, Tandem Walking, Lateral Foot Scooting to the Right, Lateral Foot Scooting to the Left, Forward to Backward Walking and Descending Stairs (Table 1; Figure 3).

The remaining CB\&M test items showed nonuniform distributions $\left(\chi^{2}\right.$-test, $\left.P<0.05\right)$, suggesting that they did not distinguish between participants. For example, Lateral Dodging and the Crouch and Walk showed a trend where most participants were given a lower score

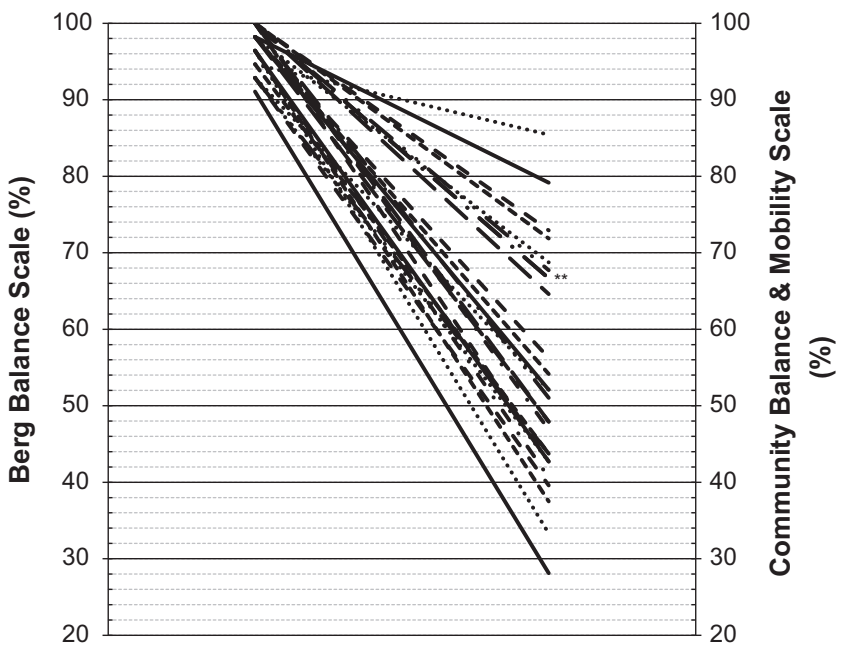

Figure 2 Performance on the BBS compared with performance on the CB\&M. Scores are expressed as a percentage of the maximum score on each measure, to facilitate comparison. Each line represents the scores of one participant. **Two participants had the same BBS and CB\&M scores. (that is, 2). In contrast, Walking and Looking, Walk, Look and Carry for Left and Right, $180^{\circ}$ Tandem Pivot and the Step-ups $\times 1$ Step Left and Right showed a trend toward having higher scores $(3,4$ or 5$)$ across participants.

\section{Convergent validity and internal consistency of the CB\&M}

Scores on the CB\&M showed a statistically significant and strong correlation with scores on the 6 MWT $(r=0.72, P<0.001)$. Scores on the CB\&M showed significant, moderately strong correlations with the $10 \mathrm{mWT}$ SS, $10 \mathrm{mWT}$ FS and BBS, respectively (10 mWT SS: $r=0.52$, $P=0.011$; 10 mWT FS: $r=0.59, P=0.004$; BBS: $r=0.47, P=0.013)$. The internal consistency of the CB\&M items was high (Cronbach's $\alpha=0.87$ ).

\section{DISCUSSION}

We investigated the use and validity of the CB\&M, a clinical measure of high-level balance, in individuals with subacute SCI receiving inpatient rehabilitation. We found that over a 7-year period the CB\&M was used by physical therapists for a small proportion of inpatients who were ambulatory (30/513 included charts). The majority of these individuals had an SCI classified as AIS D and a high LEMS. On average, the CB\&M was administered after 1 month of inpatient rehabilitation, and more specifically we observed that the CB\&M was administered once an individual had reached a high score on the BBS (that is, a score of $\geqslant 51$ ). Although all of the participants in this retrospective study had reached a near-maximal score on the BBS, their scores on the CB\&M spanned a much wider range (Figure 2), suggesting that the CB\&M does not have a ceiling effect for higherfunctioning individuals with SCI. We also found that certain test items on the CB\&M, such as Tandem Walking and Lateral Foot Scooting, appeared to discriminate between our sample of participants to a greater extent than other test items. As many CB\&M items involve challenging balance while walking, it was not surprising that CB\&M scores correlated strongly or moderately strongly with commonly used measures of walking (that is, the $6 \mathrm{MWT}$ and the $10 \mathrm{mWT}$ at

Table 1 Results from the $\chi^{2}$-test for uniform distributions

\begin{tabular}{lcr}
\hline CB\&M item & Uniform $(Y / N)$ & P-value \\
\hline Unilateral Stance Left & N & 0.014 \\
Unilateral Stance Right & Y & 0.689 \\
Tandem Walking & Y & 0.727 \\
$180^{\circ}$ Tandem Pivot & N & $<0.001$ \\
Lateral Foot Scooting Left & Y & 0.133 \\
Lateral Foot Scooting Right & Y & 0.067 \\
Hopping Forward Left & N & $<0.001$ \\
Hopping Forward Right & N & $<0.001$ \\
Crouch and Walk & N & $<0.001$ \\
Lateral Dodging & N & $<0.001$ \\
Walking and Looking Left & N & 0.001 \\
Walking and Looking Right & N & 0.001 \\
Running with Controlled Stop & N & 0.006 \\
Forward to Backward Walking & Y & 0.175 \\
Walk, Look and Carry Left & $\mathrm{N}$ & 0.013 \\
Walk, Look and Carry Right & $\mathrm{N}$ & 0.001 \\
Descending Stairs & Y & 0.37 \\
Step-ups $\times 1$ Step Left & $\mathrm{N}$ & $<0.001$ \\
Step-ups $\times 1$ Step Right & $\mathrm{N}$ & 0.001 \\
\hline Abbrition N, & &
\end{tabular}

Abbreviations: $\mathrm{N}$, no; $\mathrm{Y}$, yes.

CB\&M items with a $P$-value $>0.05$ (shaded in gray) have a uniform distribution, suggesting that performance of the participants with $\mathrm{SCl}$ on these items varied across the range of possible scores. 

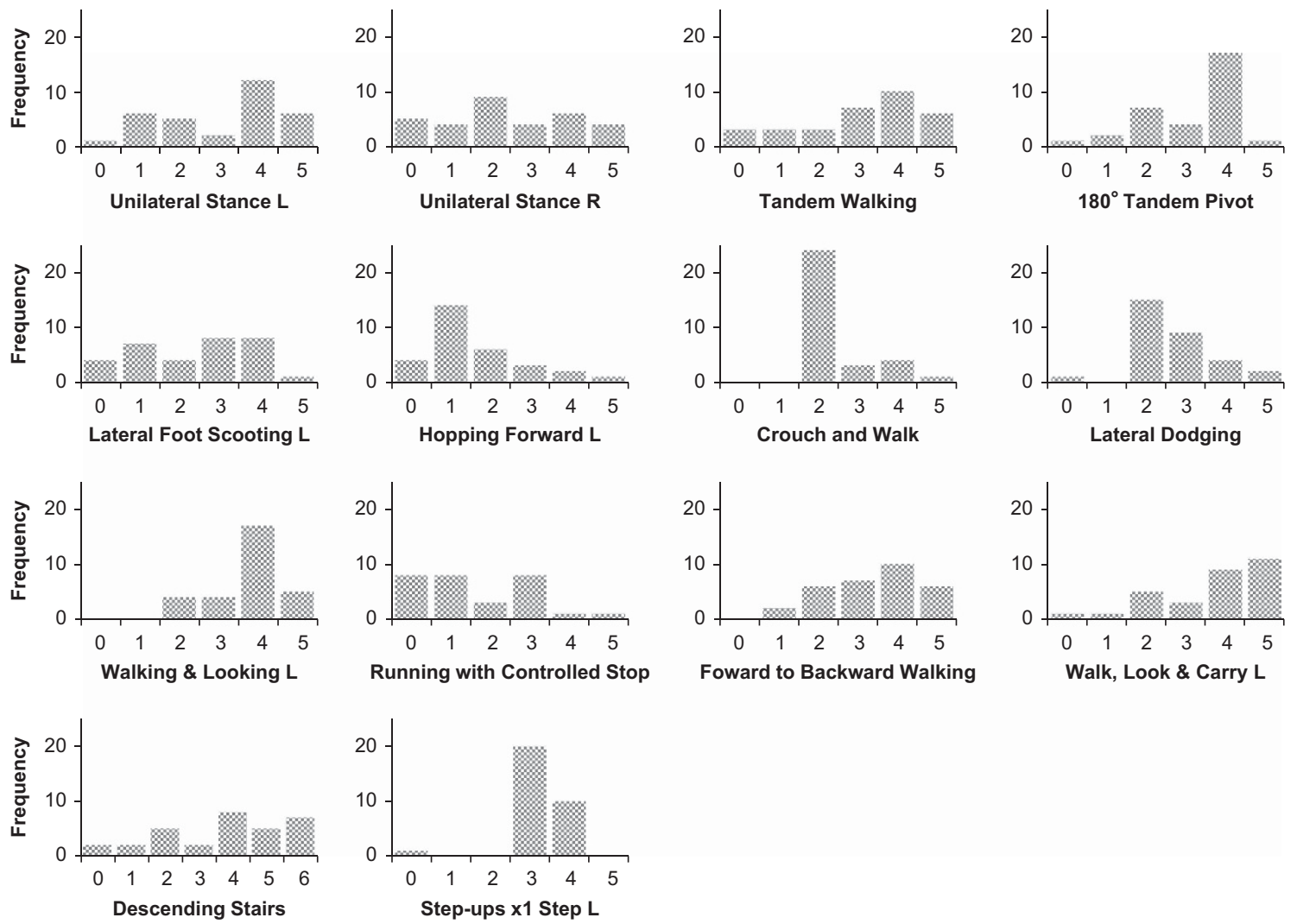

Figure 3 Histograms of scores on CB\&M items. Each item on the CB\&M is plotted separately. Only items performed on the left are plotted, except for Unilateral Stance. Both right and left items were plotted in this case, as the histograms did not look the same (plots top left corner).

self-selected and fast speeds). This demonstrates convergent validity of the CB\&M, and suggests that it may be a useful measure of walking balance. Last, the CB\&M has high internal consistency, but not sufficiently high to suggest redundancy among the test items. Collectively, our findings suggest that further study of the CB\&M in high-functioning individuals with SCI is warranted. In particular, we propose that some test items require modification to make the CB\&M more appropriate for the SCI population, and that the psychometric properties of the CB\&M for the SCI population require further investigation.

\section{Measures of walking balance for SCI}

Physical therapists and other clinicians recognize the importance of evaluating balance over the course of a patient's rehabilitation; ${ }^{24,25}$ however, the effectiveness and usefulness of these assessments are influenced by the adequacy of the assessment tools used. The majority of balance measures available to clinicians focus on sitting or standing balance (for example, BBS, Single Leg Stance Test, Functional Reach Test and so on). ${ }^{24,25}$ For example, one of the most commonly used measures of balance for SCI, the BBS, does not involve walking in any test items, but rather consists of sitting, standing and transferring activities. Yet walking is the activity resulting in the most falls in ambulatory individuals with $\mathrm{SCI}^{7}$ and this activity should be represented in measures of balance for this group. Walking tests used in the SCI population, such as the $10 \mathrm{mWT}, 6 \mathrm{MWT}$, Timed Up and Go and the Spinal Cord Injury Functional Ambulation Profile (SCIFAP),${ }^{26}$ are not appropriate indicators of walking balance. These tests allow the use of mobility aids (for example, walkers, canes), significantly reducing the stability requirements of the test.
To date there are few standardized, clinical measures that assess balance during walking tasks. The mini-BESTest is a 14-item scale that assesses six different balance control systems, such as reactive balance, sensory orientation and stability in gait, ${ }^{27}$ which has been used in the SCI population. ${ }^{28}$ This and the BBS were recently recommended, through expert consensus, as core measures of standing balance for adult populations. ${ }^{29}$ The mini-BESTest is possibly the most comprehensive balance measure currently available; ${ }^{24}$ however, the walking tasks assessed are not as challenging as those assessed in the CB\&M. As a result, there is potential for a ceiling effect. The Activitiesbased Balance Level Evaluation (ABLE) scale was developed to assess balance abilities in the SCI population throughout the full spectrum of functional recovery. ${ }^{30}$ Of the 28 items on the ABLE scale, seven involve walking; however, like the mini-BESTest the walking tasks performed are not as challenging as those encountered in the CB\&M.

\section{Potential role of the CB\&M in balance assessment for SCI}

The CB\&M addresses several of the shortcomings of other available balance measures used in individuals with SCI. First, it does not appear to show a ceiling effect like the BBS (Figure 2). This suggests that it may be a useful balance measure for higher-functioning individuals with SCI. Second, the majority of tasks on the CB\&M involve walking or behaviors similar to walking, such as running, hopping and negotiating stairs. Interestingly, CB\&M scores showed the strongest correlation with distance walked during the $6 \mathrm{MWT}$ $(r=0.72)$. The relationship between $6 \mathrm{MWT}$ distances and balance ability has been previously described in other populations. For example, in a group of individuals with mild to moderate Parkinson disease, Falvo and Earhart ${ }^{31}$ showed that 6 MWT distance was in part 
explained by balance ability (measured with the BBS) and history of falling. Similarly, in community-dwelling individuals with stroke, a strong, positive correlation was reported between $6 \mathrm{MWT}$ distance and BBS score. ${ }^{32}$ Thus, perhaps balance ability has a greater influence on $6 \mathrm{MWT}$ performance than $10 \mathrm{mWT}$ performance, a suggestion supported by the demonstration that these two walking tests must measure differing aspects of walking ability in those with SCI. ${ }^{33}$

Despite the potential advantages of the CB\&M as a balance measure, there are some drawbacks as well. First, the CB\&M is likely appropriate only for high-functioning individuals with SCI (that is, there is likely a floor effect). At our rehabilitation center, the physical therapists were selecting the CB\&M only when the inpatients achieved a near-maximal score on the BBS, and predominantly for those with an AIS D SCI and high LEMS. Indeed, the CB\&M requires performing the challenging ambulatory tasks without a walking aid. This requirement significantly limits the applicability of the scale to the broader SCI population. Second, some items on the CB\&M did not discriminate among our sample of high-functioning individuals with SCI, which causes us to question the usefulness of these test items. For example, most participants achieved high scores on the $180^{\circ}$ Tandem Pivot and Step-ups $\times 1$ Step. These or similar items are also found on the BBS, and thus it is not surprising that the participants, all of whom achieved near-perfect scores on the BBS, performed well on these tasks. In contrast, there were some items that seemed difficult for the participants, such as Hopping Forward, Crouch and Walk and Lateral Dodging. The lower-extremity strength required for these items may have exceeded that typical of individuals requiring inpatient rehabilitation. It is possible that a more uniform distribution of scores would be observed in a more chronic, community-dwelling sample of individuals with SCI. If these test items are shown to lack discrimination in a larger sample of individuals with SCI, it may warrant creation of a shortened version of the CB\&M for the SCI population.

It was surprising that Unilateral Stance on the right and Unilateral Stance on the left showed different distributions of scores among study participants. According to the $\chi^{2}$-test, the distribution of Unilateral Stance (right) showed a uniform distribution, whereas the same test item performed on the left did not. It is unlikely that leg dominance explains this finding as there is no difference between dominant and nondominant legs in the ability to perform unilateral stance in healthy adults. ${ }^{34,35}$ Upon closer inspection, six participants scored four or five points on Unilateral Stance (left), and only 0 to 2 points on the right. With the exception of one participant who had an LEMS of 28, five participants had near-normal LEMS (scores of 40, 46, 48, 49 and 50), and thus there was no notable difference in the strength of the two legs. It is possible that the sensory function (for example, proprioception) differed between the legs of these individuals; however, this could not be confirmed from the charts. The discrepancy between the results for Unilateral Stance on the right and left is likely driven by these six individuals, and likely would not persist with a larger sample size.

\section{Customizing the CB\&M for the SCI population}

Consideration should be given toward modifying the CB\&M for the SCI population. This was deduced from observations compiled by our research team, many of whom are physical therapists with many years of experience in SCI rehabilitation. Central cord syndrome and reduced upper-extremity function would limit performance on items such as the Crouch \& Walk (item six), in which a bean bag is to be picked up off the floor. Instead, we suggest simply placing a target on the floor to eliminate the grasping component of the task, but preserving the crouching component. In addition, items requiring a straight arm carry (that is, Walk, Look and Carry) pose a risk of shoulder subluxation to someone who has an injured or weakened shoulder joint. Alternative ways of carrying weight, for example an over-the-shoulder bag as used in the SCI-FAP, ${ }^{26}$ could be explored. Internal fixations in the neck and/or back may prohibit turning and looking, which is required for four CB\&M test items. Perhaps having participants fixate on a visual target in front of them when cued would be more appropriate. Last, a person with a halo would require considerable strength and motor control to bear its excess weight for items that require bending over or crouching. Developing a modified score for these individuals would be beneficial so that they are not penalized for tasks deemed unsafe.

\section{Study limitations and future directions}

This study was retrospective, and thus not all data points were available and collected for each participant. Data collection methods for the clinical measures were not standardized, potentially introducing measurement error from multiple raters. Future research directions may include investigating the relationship between falls risk and CB\&M score. We attempted to study this relationship; however, only two falls were reported during the inpatient stays of our participants. This low fall rate is probably the result of the protected environment of inpatient rehabilitation. The community-dwelling population may be a more appropriate group for the study of CB\&M performance and falls. Following customization of the CB\&M for the SCI population, we recommend that a factor analysis be completed, to identify whether or not the CB\&M items measure a single construct among the SCI population, and that the reliability (test-retest and inter-rater), validity and responsiveness of the measure be established for the SCI population. There may also be usefulness in arranging test item order according to the level of difficulty (least to most difficult). Safety may be compromised if a participant attempts a task they cannot perform and it is not anticipated by the clinician.

In conclusion, the CB\&M shows promise as a measure of high-level, ambulatory balance in individuals with incomplete SCI. Future work should focus on customizing the measure for the SCI population and evaluating its psychometric properties.

\section{DATA ARCHIVING}

There were no data to deposit.

\section{CONFLICT OF INTEREST}

The authors declare no conflict of interest.

\section{ACKNOWLEDGEMENTS}

This work was funded by the Ontario Neurotrauma Foundation.

1 Barbeau H, Nadeau S, Garneau C. Physical determinants, emerging concepts, and training approaches in gait of individuals with spinal cord injury. J Neurotrauma 2006; 23: $571-585$

2 Donnelly C, Eng JJ, Hall J, Alford L, Giachino R, Norton K et al. Client-centred assessment and the identification of meaningful treatment goals for individuals with a spinal cord injury. Spinal Cord 2004; 42: 302-307.

3 Scivoletto G, Romanelli A, Mariotti A, Marinucci D, Tamburella F, Mammone A et al. Clinical factors that affect walking level and performance in chronic spinal lesion patients. Spine 2008; 33: 259-264.

4 Brotherton SS, Krause JS, Nietert PJ. Falls in individuals with incomplete spinal cord injury. Spinal Cord 2007; 45: 37-40.

5 Amatachaya S, Wannapakhe J, Arrayawichanon P, Siritarathiwat W, Wattanapun P. Functional abilities, incidences of complications and falls of patients with spinal cord injury 6 months after discharge. Spinal Cord 2011; 49: 520-524. 
6 Wannapakhe J, Arayawichanon P, Saengsuwan J, Amatachaya S. Changes of functional ability in patients with spinal cord injury with and without falls during 6 months after discharge. Phys Ther 2014; 94: 675-681.

7 Krueger H, Noonan VK, Trenaman LM, Joshi P, Rivers CS. The economic burden of traumatic spinal cord injury in Canada. Chronic Dis Inj Can 2013; 33: 113-122.

8 Munce SEP, Wodchis WP, Guilcher SJT, Couris CM, Verrier M, Fung K et al. Direct costs of adult traumatic spinal cord injury in Ontario. Spinal Cord 2013; 51: 64-69.

9 Berg KO, Maki BE, Williams JI, Holliday PJ, Wood-Dauphinee SL. Clinical and laboratory measures of postural balance in an elderly population. Arch Phys Med Rehabil 1992; 73: 1073-1080.

10 Datta S, Lorenz DJ, Harkema SJ. Dynamic longitudinal evaluation of the utility of the Berg Balance Scale in individuals with motor incomplete spinal cord injury. Arch Phys Med Rehabil 2012; 93: 1565-1573.

11 Lemay JF, Nadeau S. Standing balance assessment in ASIA D paraplegic and tetraplegic participants: concurrent validity of the Berg Balance Scale. Spinal Cord 2010; 48: 245-250.

12 Lapointe R, Lajoie Y, Serresse O, Barbeau H. Functional community ambulation requirements in incomplete spinal cord injured subjects. Spinal Cord 2001; 39 : 327-335.

13 Inness EL, Howe J, Niechwiej-Szwedo E, Jaglal SB, Mcllroy WE, Verrier MC. Measuring balance and mobility after traumatic brain injury: validation of the Community Balance and Mobility Scale (CB\&M). Physiother Can 2011; 63: 199-208.

14 Wright FV, Ryan J, Brewer K. Reliability of the Community Balance and Mobility Scale (CB\&M) in high-functioning school-aged children and adolescents who have an acquired brain injury. Brain Injury 2010; 24: 1585-1594.

15 Balasubramanium CK. The Community Balance and Mobility Scale alleviates the ceiling effects observed in the currently used gait and balance assessments for the community-dwelling older adults. J Geriatr Phys Ther 2015; 38: 78-89.

16 Wright MJ, Bos C. Performance of children on the Community Balance and Mobility Scale. Phys Occup Ther Pediatr 2012; 32: 416-429.

17 Knorr S, Brouwer B, Garland SJ. Validity of the Community Balance and Mobility Scale in community-dwelling persons after stroke. Arch Phys Med Rehabil 2010; 91: 890-896.

18 Takacs J, Garland J, Carpenter MG, Hunt MA. Validity and reliability of the Community Balance and Mobility Scale in individuals with knee osteoarthritis. Phys Ther 2014; 94: 866-874.

19 Bos C. A new clinical tool to measure balance and mobility in hemophilia: the Community Balance and Mobility Scale. Haemophilia 2012; 18: 142.

20 Guyatt GH, Sullivan MJ, Thompson PJ, Fallen EL, Pugsley SO, Taylor W et al. The 6-minute walk: a new measure of exercise capacity in patients with chronic heart failure. Can Med Assoc J 1985; 132: 919-923.
21 van Hedel HJ, Wirz M, Dietz V. Assessing walking ability in subjects with spinal cord injury: validity and reliability of 3 walking tests. Arch Phys Med Rehabil 2005; 86 190-196.

22 Evans JD. Straightforward Statistics for the Behavioral Sciences. Brooks/Cole Publ. Co.: Pacific Grove, California, USA, 1996.

23 Streiner DL, Norman GR. Health Measurement Scales: A Practical Guide to their Development and Use. Oxford University Press: Toronto, ON, Canada, 2008.

24 Sibley KM, Straus SE, Inness EL, Salbach NM, Jaglal SB. Balance assessment practices and use of standardized balance measures among Ontario physical therapists. Phys Ther 2011; 91: 1583-1591.

25 Sibley KM, Straus SE, Inness EL, Salbach NM, Jaglal SB. Clinical balance assessment: perceptions of commonly-used standardized measures and current practices among physiotherapists in Ontario, Canada. Implement Sci 2013; 8: 33.

26 Musselman KE, Brunton K, Lam T, Yang J. Spinal cord injury functional ambulation profile: a new measure of walking ability. Neurorehabil Neural Repair 2011; 25: 285-293.

27 Franchignoni F, Horak F, Godi M, Nardone A, Giordano A. Using psychometric techniques to improve the balance evaluation system's test: the mini-BESTest. J Rehabil Med 2010; 42: 323-331.

28 Lemay JF, Gagnon D, Duclos C, Grangeon M, Gauthier C, Nadeau S. Influence of visual inputs on quasi-static standing postural steadiness in individuals with spinal cord injury. Gait Posture 2013; 38: 357-360.

29 Sibley KM, Howe T, Lamb SE, Lord SR, Maki BE, Rose DJ et al. Recommendations for a core outcome set for measuring standing balance in adult populations: a consensusbased approach. PLOS ONE 2015; 10: e0120568.

30 Ardolino EM, Hutchinson KJ, Pinto Zipp G, Clark M, Harkema SJ. The ABLE Scale: The development and psychometric properties of an outcome measure for the spinal cord injury population. Phys Ther 2012; 92: 1046-1054.

31 Falvo MJ, Earhart GM. Six-minute walk distance in persons with Parkinson disease a hierarchical regression model. Arch Phys Med Rehabil 2009; 90: 1004-1008.

32 Eng JJ, Chu KS, Dawson AS, Kim CM, Hepburn KE. Functional walk tests in individuals with stroke. Stroke 2002; 33: 756-761.

33 Forrest GF, Hutchinson K, Lorenz DJ, Buehner JJ, VanHiel LR, Sisto SA et al. Are the 10 meter and 6 minute walk tests redundant in patients with spinal cord injury? PLoS ONE 2014; 9: e94108.

34 Hoffman M, Schrader J, Applegate T, Koceja D. Unilateral postural control of the functionally dominant and nondominant extremities of healthy subjects. J Athl Train 1998; 33: 319-322.

35 Cug M, Ozdemir RA, Emre AK. Influence of leg dominance on single-leg stance performance during dynamic conditions: an investigation into the validity of symmetry hypothesis for dynamic postural control in healthy individuals. Turk J Phys Med Rehab 2014; 60: 22-26. 